\title{
MUC1, MUC2, MUC5AC, and MUC6 in colorectal cancer: expression profiles and clinical significance
}

\author{
Johannes Betge $^{1}$ • Nora I. Schneider ${ }^{2} \cdot$ Lars Harbaum $^{3} \cdot$ Marion J. Pollheimer $^{2}$ • \\ Richard A. Lindtner ${ }^{4} \cdot$ Peter Kornprat $^{5} \cdot$ Matthias P. Ebert $^{1} \cdot$ Cord Langner $^{2}$
}

Published online: 14 June 2016

(C) The Author(s) 2016. This article is published with open access at Springerlink.com

\begin{abstract}
Mucin glycoprotein expression can be altered during the carcinogenic process. The impact on the prognosis of patients with colorectal cancer (CRC) is controversial. We analyzed tumors from 381 patients for MUC1, MUC2, MUC5AC, and MUC6 expression by immunohistochemical staining, using tissue microarrays. Progression-free and cancer-specific survival were determined using the KaplanMeier method. Expression of intestinal mucin MUC2 was lost in $85(23 \%)$ CRCs, and patients with MUC6-negative tumors showed shorter progression-free survival (PFS, $p=0.043$ ). Gastric mucins MUC5AC and MUC6 showed high (>50\%) aberrant expression in $28(8 \%)$ and $9(2 \%)$ cases, respectively. High expression of MUC5AC was associated with longer PFS $(p=0.055)$. High expression of MUC6 was associated with $100 \%$ PFS $(p=0.024)$ and longer cancer-specific survival (CSS, $p=0.043)$. MUC1 was expressed in $238(64 \%)$ tumors and had no impact on outcome. When analysis was restricted to stages II and III, loss of MUC2 was associated with adverse outcome. Overexpression of both MUC5AC and MUC6 significantly predicted favorable PFS and CSS.
\end{abstract}

Cord Langner

cord.langner@medunigraz.at

1 Department of Medicine II, University Hospital Mannheim, Medical Faculty Mannheim: Heidelberg University, Mannheim, Germany

2 Institute of Pathology, Medical University of Graz, Auenbruggerplatz 25, 8036 Graz, Austria

3 Department of Medicine II, University Medical Center Hamburg-Eppendorf, Hamburg, Germany

4 Department of Surgery, Medical University of Innsbruck, Innsbruck, Austria

5 Department of Surgery, Division of General Surgery, Medical University of Graz, Graz, Austria
In conclusion, loss of MUC2 expression proved to be a predictor of adverse outcome, while the gain of aberrant expression of MUC5AC and particularly of MUC6 was associated with favorable outcome in CRC, notably in intermediate stages II and III.

Keywords Mucin $\cdot$ Prognosis $\cdot$ Stage II ·

Immunohistochemistry $\cdot$ Large intestine $\cdot$ Prognostic factor

\section{Introduction}

Tumor stage reflected by the AJCC/UICC TNM system is currently the strongest prognostic parameter for patients with colorectal cancer (CRC) and is therefore used as basis for therapeutic decisions [1]. However, patients with tumors of the same pathologic stage may experience substantially different clinical outcomes, especially in intermediate stages of disease. Different patients may thus benefit from different therapeutic and surveillance strategies [2, 3]. Hence, identification of additional prognostic markers might be of interest to improve stratification of patients.

Mucins are high-molecular-weight glycoproteins expressed by epithelial tissues. They have a high content of clustered oligosaccharides, forming a mucosal protection system at the surface of the gastrointestinal tract [4]. Most of the recent progress in understanding mucin biology is related to the characterization of mucin proteins (apomucins) [5]. Mucins can be classified as secreted gel-forming mucins (MUC2, MUC5AC, and MUC6), transmembrane mucins (MUC1), and other mucins that do not fit into either class [5, 6]. Throughout the gastrointestinal tract, epithelial cells commonly synthesize more than one mucin, although a particular mucin type may predominate in an organ-specific manner. For example, MUC2 is characteristically observed in goblet cells 
of small and large bowel mucosa, whereas MUC5AC and MUC6 are mainly expressed in gastric epithelium and are rarely observed in the normal colon [5-7]. In contrast, MUC1 is expressed on the apical surface of most epithelial cells [8]. Of note, during neoplastic transformation and/or progression, expression of specific mucins may be reduced or the organ specificity lost, while new mucins may be expressed aberrantly.

In colorectal tumors, several mucins have been analyzed, in relation to the adenoma-carcinoma sequence, MUC1 and MUC2 being the best characterized [4, 6-9]. However, data on the clinical significance, particularly the potential prognostic value of mucin expression in colorectal carcinoma (CRC) are limited and contradictory $[5,6,9,10]$.

Therefore, our study aimed to comprehensively assess the prevalence of MUC1, MUC2, MUC5AC, and MUC6 expression in a large cohort of CRC patients, its association with various clinicopathological parameters such as $\mathrm{T}$ classification, $\mathrm{N}$ classification, lymphovascular invasion, grade, tumor border configuration, mismatch repair (MMR) status, as well as patients' progression-free and cancer-specific survival.

\section{Patients and methods}

\section{Case selection}

Case selection of our cohort has been described in detail previously [11]. Briefly, 400 CRC patients treated from January 1992 through December 2000 were randomly selected from the CRC database of the Institute of Pathology, Medical University of Graz, Austria. We excluded (1) patients with T1 cancer treated by endoscopic polypectomy, (2) patients that received neoadjuvant chemotherapy, (3) patients with synchronous or metachronous invasive cancers originating from the colorectum or other sites. In total, 381 resection specimens from 400 patients ( $95 \%$ ) were available for pathology review. Of these, 215 were males (56\%) and 166 females (44\%) (ratio 1.3:1) with a mean age of 68.5 years (median 70.1 , range 27.6-93.1).

Stage I and II patients did not receive adjuvant therapy, while stage III patients were treated with 5-fluorouracil/folic acid according to the Mayo Clinic regimen [12]. Patients with node-positive rectal cancer received adjuvant radiotherapy. Follow-up chest X-ray and abdominal ultrasound were performed at 6-month intervals for the first 3 years and yearly thereafter. Moreover, laboratory testing (blood count, liver enzymes, CEA, and CA19-9) was performed at 3-month intervals for the first 3 years and 6-month intervals thereafter. Patients with rectal cancer underwent pelvic computerized tomography every 12 months. Patients were followed after resection until death or time of last follow-up. Disease progression was defined as local tumor recurrence or development of distant metastasis.

\section{Pathological evaluation}

Original histopathological slides were independently reevaluated by two gastrointestinal pathologists (M.J.P. and C.L.). Tumors located in the cecum and transverse colon were defined as right-sided cancers, while tumors located in the left colonic flexure down to the sigmoid colon were defined as left-sided cancers. Tumors located at the rectosigmoid junction or in the rectum were defined as rectal cancers. Tumor stage was assessed according to the AJCC/UICC 2009 issue of the TNM classification [13]. Histological tumor type and tumor grade were established according to the WHO guidelines [14]. The presence of lymph and/or blood vessel invasion was assessed as carcinoma cells present in vessels with an unequivocal endothelial lining (lymphatic invasion) or in vessels with a thick vascular wall and red blood cells in the lumen (blood vessel invasion). Tumor border configurations were evaluated according to the Jass classification (expanding vs infiltrative) [15]. The extent of tumor budding (presence of isolated single cells or small clusters of tumor cells in the stroma at the invasive tumor margin) was assessed on H\&Estained slides in a field in which budding intensity was maximal [16]. The number of budding foci was scored as low grade $(<10$ budding foci) or high grade ( $>9$ budding foci) [17].

\section{Immunohistochemistry}

A tissue microarray technique was used for immunohistochemical evaluation as described previously [18]. Briefly, tissue microarrays (TMAs) were constructed using a manual tissue-arraying instrument (Beecher, Silver Spring, MD, USA). Between 3 and 14 (mean 5.03, median 5) cylindrical core biopsies, $0.6 \mathrm{~mm}$ in diameter, were taken from different sites of each tumor and arrayed in a recipient paraffin TMA.

For immunohistichemical staining, $4 \mu \mathrm{m}$ TMA sections were treated with $1 \% \mathrm{H}_{2} \mathrm{O}_{2}$, subjected to antigen retrieval and subsequently incubated for $30 \mathrm{~min}$ with primary antibodies using automated staining systems (Universal Staining System, DakoCytomation, Glostrup, Denmark, or BenchMark, Ventana Medical Systems, S.A, Illkirch CEDEX, France). Details on primary antibodies, dilution, and epitope retrieval are listed in Table 1. The reaction was visualized by the Dako EnVision system detection kit, or the ultraVIEW Universal DAB detection kit (Ventana). Goblet cells in adjacent non-neoplastic colonic mucosa served as positive control for MUC2, slides of gastric cancer, known to express MUC1, MUC5AC, and MUC6 served as positive control for MUC1, MUC5AC, and MUC6. Negative controls included omission of the primary antibodies and incubation with Dako ChemMate Antibody Diluent (code 
Table 1 Antibodies used for immunohistochemical staining

\begin{tabular}{llllll}
\hline Antibody & Clone & Dilution & Epitope-retrieval & Chromogen & Source \\
\hline MUC1 & Ma695 & $1: 100$ & HIER & DAB & Novocastra, Newcastle upon Tyne, UK \\
MUC2 & Ccp58 & $1: 50$ & HIER & DAB & Novocastra \\
MUC5AC & 45 M1 & $1: 100$ & MW, Tris-HCl urea & AEC & Abnova, Taipei, Taiwan \\
MUC6 & MCN6.01 & $1: 100$ & MW, Tris-HCl urea & AEC & Neomarker, CA, USA \\
MLH1 & G168-15 & $1: 50$ & MW, buffer pH 9 & DAB & Biocare, Concorde, CA, USA \\
MSH2 & G219-1129 & $1: 50$ & Buffer CC1 standard & DAB & Ventana, Tucson, AZ, USA \\
MSH6 & BC-44 & $1: 50$ & Buffer CC1 mild & DAB & Biocare \\
\hline
\end{tabular}

AEC 3-amino-9-ethylcarbazole, buffer pH 9 target retrieval solution, Dako (S2367), CC1 cell conditioning 1 (Ventana 950-124 SL), DAB 3,3'-diaminobenzidine, HIER heat induced epitope retrieval solution (Dako, code no. K 5205), 40 min $98{ }^{\circ} \mathrm{C}, M W$ microwave

no. S 2022) or Ventana Antibody Diluent (catalog no. 251018), respectively.

Immunoreactivity of MUC1, MUC2, MUC5AC, and MUC6 was assessed by two investigators (J.B. and N.I.S.), who were blinded to clinicopathologic data. Distinct membranous and/or granular cytoplasmic staining was considered positive. Immunoreactivity was semiquantitatively categorized as "negative" (0\% of tumor cells positive) as "low" ( $<50 \%$ of tumor cells positive) or as "high" ( $>50 \%$ of tumor cells positive). Each tumor was scored by assessing the average immunoreactivity of the TMA cores. For validation of staining results obtained from TMA slides, we performed immunohistochemistry on corresponding whole sections in selected cases (compare below).

MMR status was assessed as described earlier, using antibodies directed against MLH1, MSH2, and MSH6 [18]. The loss of immunoreactivity for at least one of the three markers characterized MMR-deficient tumors [19].

\section{Statistical analysis}

Associations with $\mathrm{T}$ classification, $\mathrm{N}$ classification, AJCC/UICC stage, grade, lymphovascular invasion, MMR status, location, tumor type, tumor budding, and tumor border configuration were analyzed using the chi-squared test or Fisher's exact test. Cause of death was determined by treating physicians and/or by chart review and was corroborated by death certificates if available. Progression-free/disease-free (PFS) and cancer-specific survival (CSS) were investigated using the Kaplan-Meier method and compared by the logrank test. For bivariate testing, Cox proportional hazards regression models were performed. Statistical calculations were performed using $\mathrm{R}$ version 3.2.0 ( $\mathrm{R}$ Foundation for Statistical Computing, Vienna, Austria; http://www.Rproject.org/) or with SPSS version 20.0 (IBM, Armonk, NY, USA). All reported $p$ values were two-sided with significance at $p<0.05$.

\section{Results}

\section{Tumor characteristics}

We classified $28(7 \%)$ tumors as pT1, $70(18 \%)$ as pT2, 218 $(57 \%)$ as pT3, and $65(17 \%)$ as pT4. Lymph node metastases were detected in 165 (43\%) cases. Tumor grades were G1 in 121 (32\%), G2 in 138 (36\%), and G3 in 122 (32\%) cases. Regarding tumor type, 317 (83\%) cases were adenocarcinomas and $44(12 \%)$ were mucinous adenocarcinomas. The remaining tumors presented with rare histological subtypes such as 13 (3\%) undifferentiated carcinomas, 3 signet-ring cell, 2 medullary, 1 amphicrine, and 1 adenosquamous carcinoma. Lymphovascular invasion was recorded in 158 (41\%) tumors.

High expression of MUC1 was observed in 46 (12\%) and low expression in 192 (52\%) tumors, while 134 (36\%) tumors were negative for MUC1 (Fig. 1a). High expression of MUC2 was observed in 61 (16\%) and low expression in $225(61 \%)$ tumors, while expression of MUC2 was lost in $85(23 \%)$ cases (Fig. 1b). High aberrant expression of MUC5AC was observed in $28(8 \%)$ and low expression in $155(42 \%)$ tumors, while $189(51 \%)$ tumors were negative for MUC5AC (Fig. 1c). High expression of MUC6 was observed in $9(2 \%)$ and low expression in $99(27 \%)$ tumors, while 264 (71 \%) lacked MUC6 expression (Fig. 1d).

Expression of MUC1 was significantly associated with $\mathrm{T}$ and N classification, AJCC/UICC stage, and tumor differentiation and inversely correlated with mucinous adenocarcinoma histological type. MUC2, MUC5AC, and MUC6 were significantly associated with MMR status and tumor border configuration. Additionally, MUC2 expression was associated with mucinous differentiation and inversely correlated with lymphovascular invasion and tumor differentiation. MUC5AC expression correlated with tumor location and mucinous subtype. MUC6 inversely correlated with tumor differentiation and mucinous adenocarcinoma subtype (Table 2). MUC5AC expression significantly correlated with MUC2 
Fig. 1 Examples of immunohistochemical staining of MUC1 $(\times 200, \mathbf{a}), \operatorname{MUC2}(\times 200$, b), MUC5AC $(\times 200, \mathbf{c})$, and MUC6 $(\times 200$, d) using TMA slides
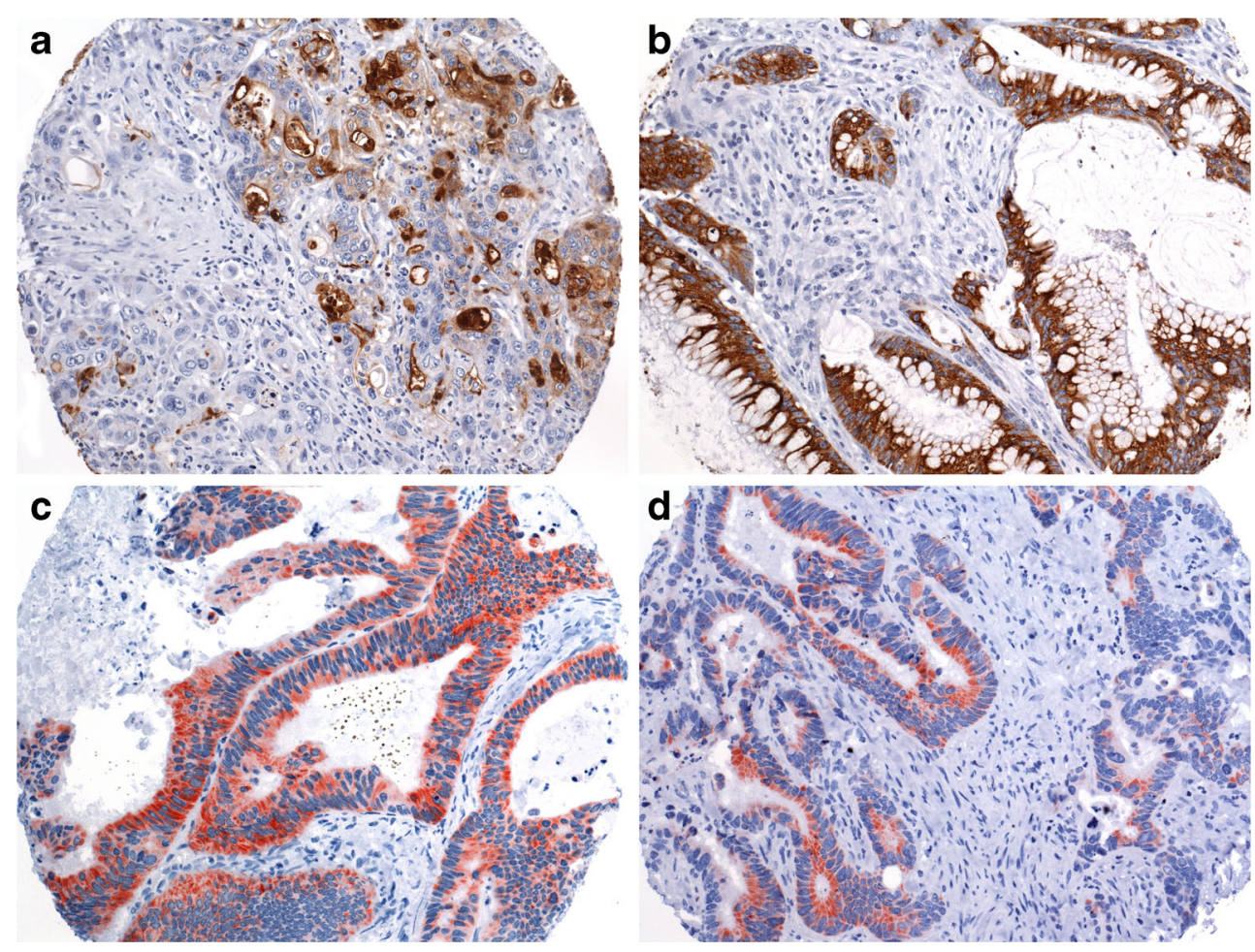

and MUC6, while MUC6 expression significantly correlated with expression of MUC1 and MUC5AC (Table 3).

For validation of TMA staining results, a subset of cases with negative and low and high expressions of MUC1, MUC2, MUC5AC, and MUC6 was analyzed on corresponding whole sections. Staining results obtained from TMA slides were confirmed in all analyzed cases (Fig. 2).

\section{Survival analysis}

For 350 out of 381 (92\%) patients, follow-up data were available. Progressive disease was observed in 141 (40\%) patients after a median (mean) follow-up of 45 months (56) (range 1-182). Eleven patients were alive with metastatic disease at the end of follow-up, 118 (34\%) patients died from cancer. Seven patients were without evidence of disease after metastasectomy. Five patients that had presented in poor condition due to advanced disease died within 30 days of surgery. Mean time to progression was 15 months (median 7, range $0-88$ ) [11].

\section{MUC1}

Disease progression occurred in $43 \%$ of patients with tumors positive (high or low) for MUC1, compared with $36 \%$ of patients with tumors negative for MUC1 $(p=0.2)$. Actuarial 5-year PFS rates were 56 and $64 \%$, respectively. In addition, $35 \%$ of patients with MUC1positive and $32 \%$ of patients with MUC1-negative tumors died of disease $(p=0.48)$. Actuarial 5-year CSS rates were 63 and $67 \%$, respectively.

\section{$M U C 2$}

Disease progression occurred in $52 \%$ of patients with tumors negative for MUC2 compared with $37 \%$ of patients with tumors positive (high or low) for MUC2 $(p=0.043$; Fig. 3a). Actuarial 5-year PFS rates were 50 and $62 \%$, respectively. In addition, $42 \%$ of patients with MUC2-negative and $32 \%$ of patients with MUC2-positive tumors died of disease $(p=0.15$; Fig. 3b). Actuarial 5 -year CSS rates were 60 and $66 \%$, respectively.

\section{MUC5AC}

Disease progression occurred in $37 \%$ of patients with tumors positive (high or low) for MUC5AC, compared with $44 \%$ of patients with tumors negative for MUC5AC $(p=0.46)$. Actuarial 5-year PFS rates were 63 and $56 \%$, respectively. In addition, $30 \%$ patients with MUC5AC-positive and $44 \%$ of patients with MUC5AC-negative tumors died of disease $(p=0.28)$. Actuarial 5-year CSS rates were 67 and $62 \%$, respectively. Of note, the extent of MUC5AC expression was found to be related to disease outcome, that is, only $20 \%$ of patients with high ( $>50 \%$ of tumor cells) MUC5AC expression experienced disease progression, compared with $42 \%$ of patients with low or absent MUC5AC expression ( $p=0.055$; Fig. $3 c)$. Actuarial 5-year PFS rates 


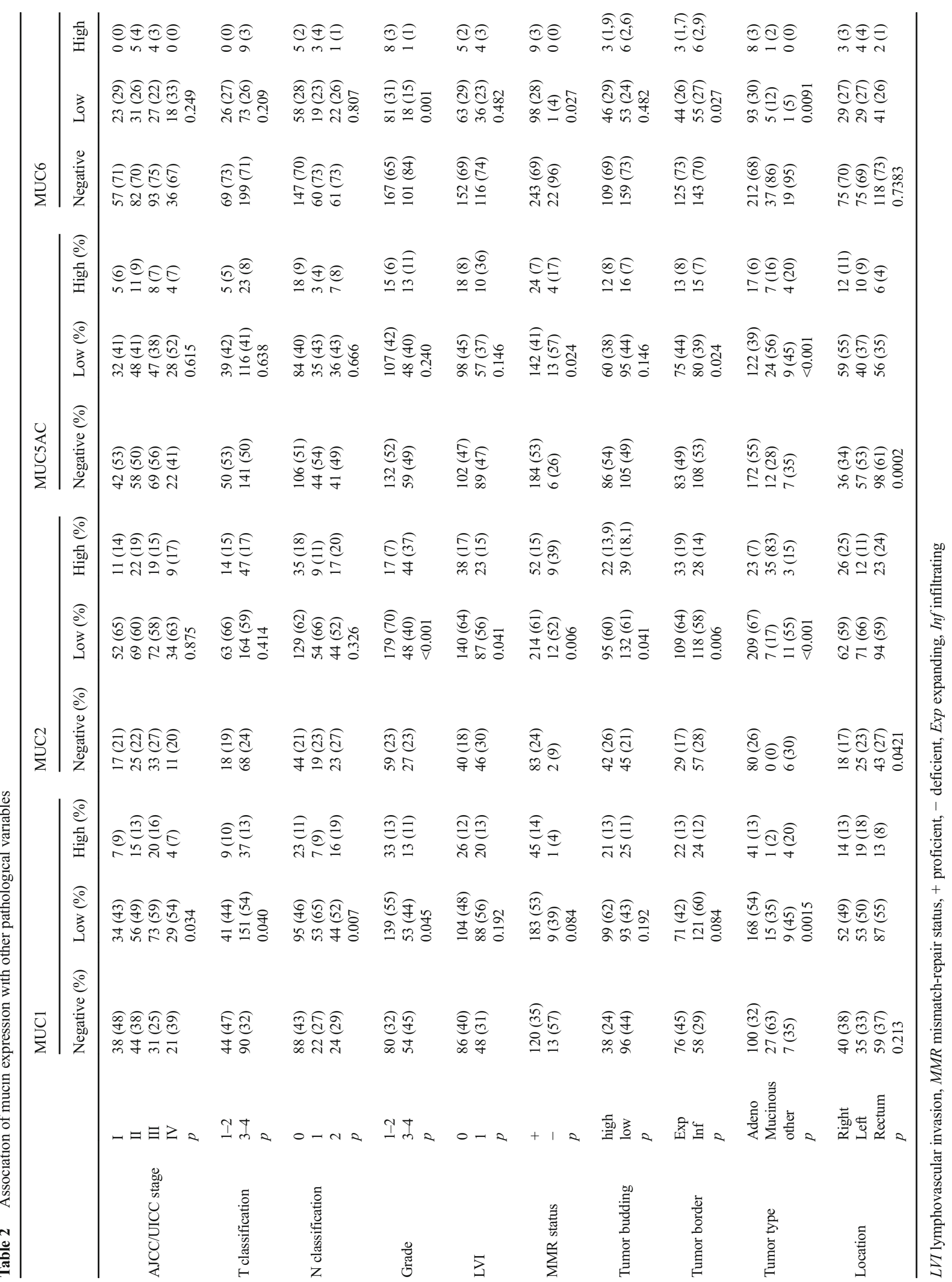


Table 3 Interrelations of different mucins expressed in CRC

\begin{tabular}{|c|c|c|c|c|c|c|c|c|c|c|}
\hline & & \multicolumn{3}{|l|}{ MUC2 } & \multicolumn{3}{|l|}{ MUC5AC } & \multicolumn{3}{|l|}{ MUC6 } \\
\hline & & Negative $(\%)$ & Low (\%) & High $(\%)$ & Negative (\%) & Low $(\%)$ & High (\%) & Negative (\%) & Low $(\%)$ & High (\%) \\
\hline \multirow{12}{*}{ MUC1 } & 0 & $33(25)$ & $71(53)$ & $29(22)$ & $77(58)$ & $49(37)$ & $8(6)$ & $119(89)$ & $15(11)$ & $0(0)$ \\
\hline & $<50 \%$ & $43(22)$ & $120(63)$ & $29(15)$ & $92(48)$ & $87(45)$ & $13(7)$ & $120(63)$ & $69(36)$ & $3(2)$ \\
\hline & $>50 \%$ & $9(20)$ & $34(74)$ & $3(7)$ & $20(44)$ & $19(41)$ & $7(15)$ & $25(54)$ & $15(33)$ & $6(13)$ \\
\hline & \multirow[t]{9}{*}{$p$} & & 0.0797 & & & 0.106 & & & 0.001 & \\
\hline & & & & 0 & $66(77)$ & $18(21)$ & $2(2)$ & $76(88)$ & $9(11)$ & $1(1)$ \\
\hline & & \multirow{7}{*}{ MUC2 } & & $<50 \%$ & $109(48)$ & $104(46)$ & $13(6)$ & $143(63)$ & $77(34)$ & $7(3)$ \\
\hline & & & & $>50 \%$ & $16(26)$ & $32(53)$ & $13(21)$ & $47(77)$ & $13(21)$ & $1(2)$ \\
\hline & & & & $p$ & & 0.001 & & & 0.001 & \\
\hline & & & & & & & 0 & $178(93)$ & $13(7)$ & $0(0)$ \\
\hline & & & & & \multirow{3}{*}{ MUC5AC } & & $<50 \%$ & $75(48)$ & $79(51)$ & $1(1)$ \\
\hline & & & & & & & $>50 \%$ & $13(46)$ & $7(25)$ & $8(29)$ \\
\hline & & & & & & & $p$ & & 0.001 & \\
\hline
\end{tabular}

were 79 and $58 \%$, respectively. In addition, $20 \%$ of patients with high MUC5AC expression and $38 \%$ of patients with low or absent MUC5AC expression died of disease ( $p=0.18$; Fig. 3d). Actuarial 5-year CSS rates were 77 and $64 \%$, respectively.

\section{MUC6}

Disease progression occurred in $39 \%$ of patients with tumors positive (high or low) for MUC6, compared with
99 out of $243(41 \%)$ patients with tumors negative for MUC6 $(p=0.83)$. Actuarial 5-year PFS rates were 61 and $59 \%$, respectively. In addition, $30 \%$ of patients with MUC6-positive and $35 \%$ of patients with MUC6-negative tumors died of disease $(p=0.25)$. Actuarial 5-year CSS rates were 70 and $62 \%$, respectively. Again, the extent of expression was found to be related to disease outcome, that is, disease progression occurred in $41 \%$ of patients with low or absent MUC6 expression, while none of the nine patients with high MUC6 expression experienced
Fig. 2 Examples of TMA validation using whole sections (showing identical staining results): low expression of MUC1 $(\times 100$, a), low expression of MUC2 $(\times 100, \mathbf{b})$, high expression of MUC2 $(\times 40, \mathbf{c})$, and negative staining for MUC5AC $(\times 40, d)$. Roundish tissue defects owing to punch-out of tumor for TMA generation are included in all images
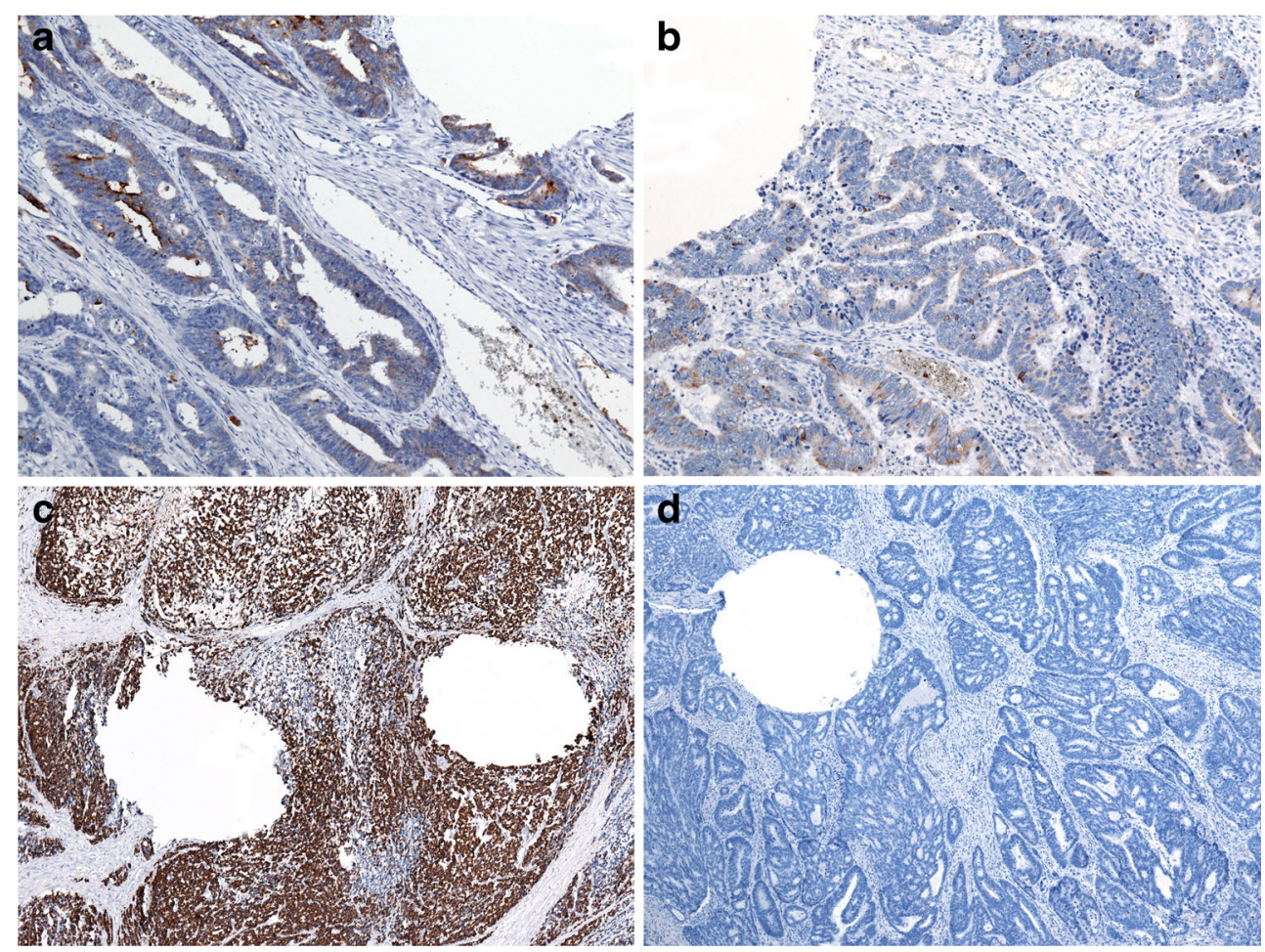
a

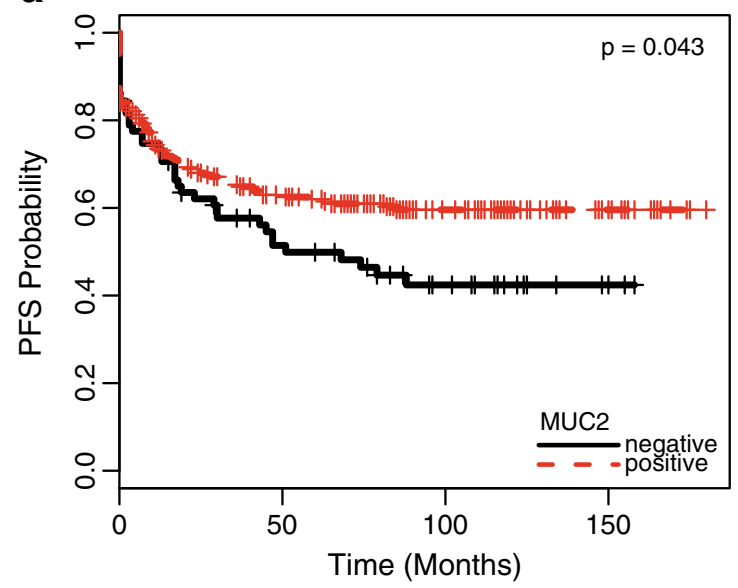

C

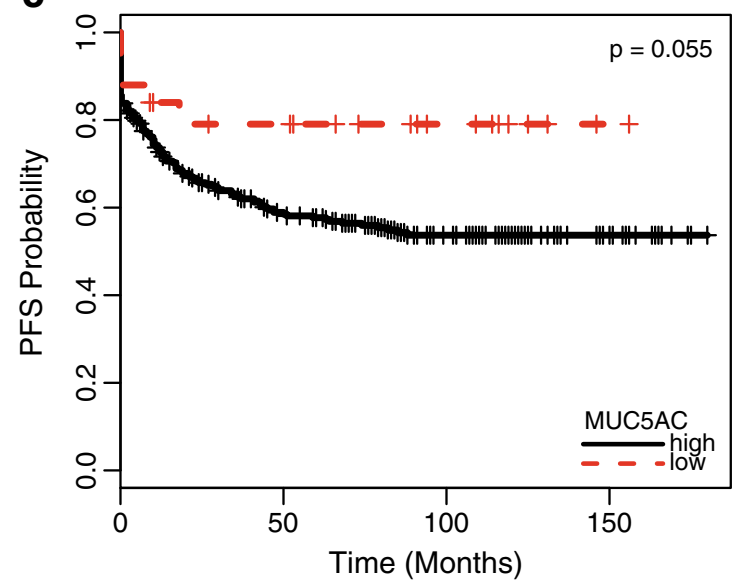

e

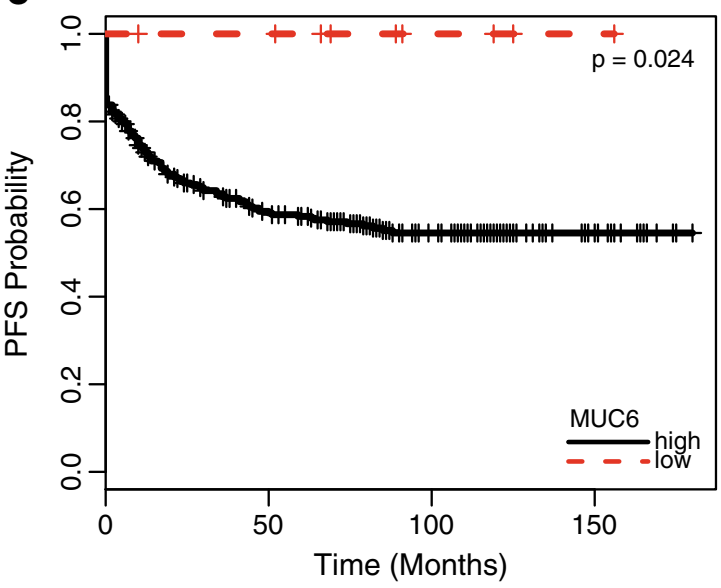

Fig. 3 Progression-free (a, $p=0.043)$ and cancer-specific $(\mathbf{b}, p=0.15)$ survival of patients with colorectal cancer related to the extent of MUC2 expression (present vs. absent). Progression-free (c, $p=0.055)$ and cancer-specific (c, $p=0.18$ ) survival of patients with colorectal cancer

progressive disease ( $p=0.024$; Fig. 3e). Actuarial 5-year PFS rates were 100 and $58 \%$, respectively. In addition, $34 \%$ of patients with low or absent MUC6 expression and b

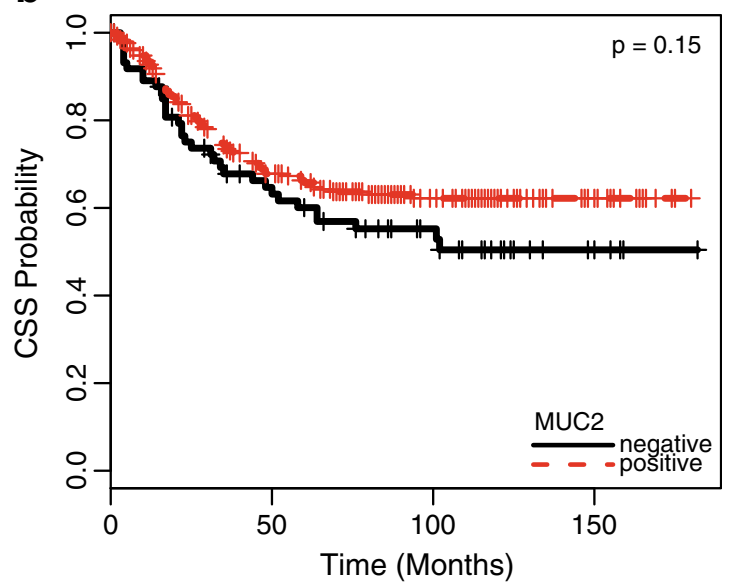

d

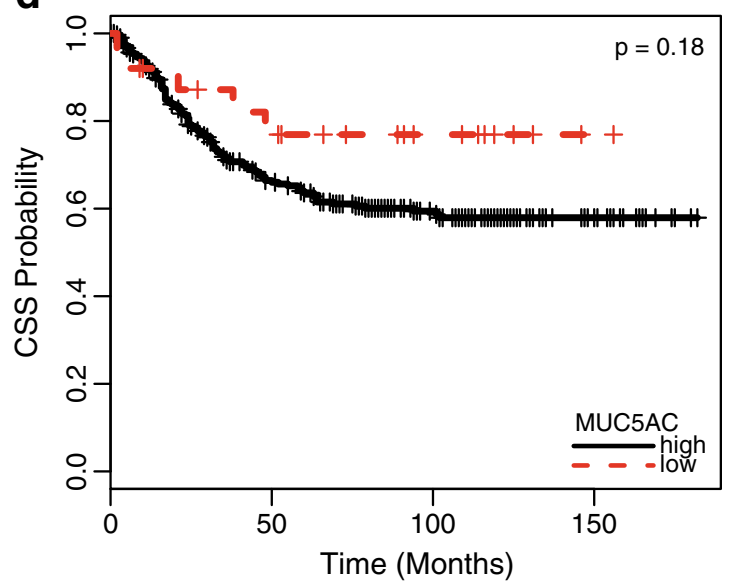

f

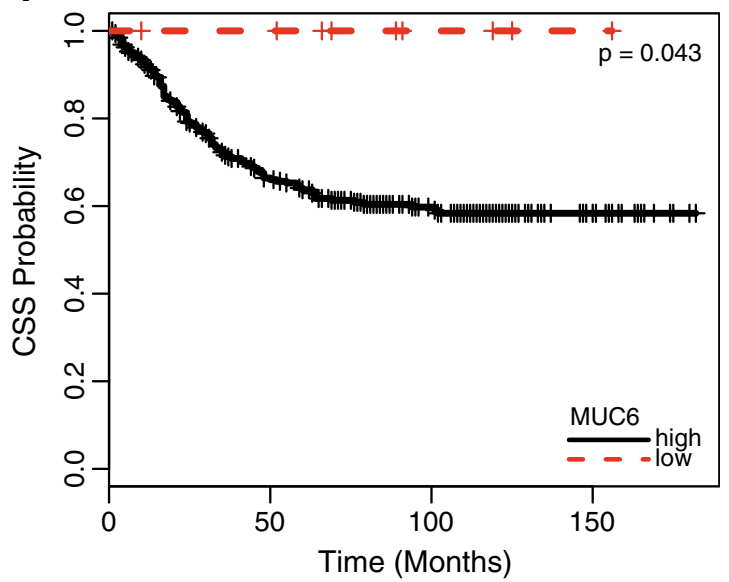

related to the extent of MUC5AC expression (high vs. low expression). Progression-free (e, $p=0.024)$ and cancer-specific $(\mathbf{f}, p=0.043)$ survival of patients with colorectal cancer related to the extent of MUC6 expression (high vs. low expression)

no patient with high MUC6 expression died of disease ( $p=0.043$; Fig. 3f). Actuarial 5-year CSS rates were 100 and $64 \%$, respectively. 


\section{Combined analysis}

Accordingly, patients with tumors that showed either preserved MUC2 expression and/or high expression of MUC5AC and/or a high expression of MUC6 had longer PFS $(p=0.031)$ but not CSS $(p=0.11)$ than patients lacking those features.

\section{Stages II and III}

Loss of intestinal mucin (MUC2) was also related to shorter PFS ( $p=0.003$, Fig. 4a) and CSS ( $p=0.089$, Fig. 4b), when analysis was restricted to patients with intermediate stages of disease, that is, AJCC/UICC stages II and III. Likewise, gain of gastric mucin (MUC5AC and MUC6) was related to improved outcome. Specifically, high MUC5AC expression predicted favorable PFS ( $p=0.015$, Fig. $4 c)$ and CSS ( $p=0.033$, Fig. 4d), as did high MUC6 expression (PFS: $p=0.03$, Fig. 4e; CSS: $p=0.047$, Fig. 4f). Finally, patients with stage II and III disease with tumors that showed either preserved MUC2 expression and/or high expression of MUC5AC and/ or a high expression of MUC6 had longer PFS ( $p=0.0018)$ and CSS $(p=0.062)$ than patients lacking those features.

\section{MMR-positive and MMR-negative patients}

Since MMR expression is known to be a confounder of mucin expression, we analyzed MMR positive and negative patients separately. In patients with MMR proficient tumors $(n=350)$, loss of MUC2 was associated with shorter PFS $(p=0.024)$ and CSS $(p=0.095)$. High expression of MUC5AC was related to shorter PFS $(p=0.047)$, while the association of MUC5AC with decreased CSS was not statistically significant $(p=0.12)$. High expression of MUC6 was associated with decreased PFS $(p=0.023)$ and $\operatorname{CSS}(p=0.042)$. MUC1 expression showed no impact on PFS $(p=0.28)$ or CSS $(p=0.65)$. In patients with MMR-deficient tumors ( $n=23)$, no significant impact on either PFS or CSS was observed.

Additionally, we performed bivariate Cox proportional hazards regression models including MMR status and mucin expression. MUC2 negativity was significantly associated with PFS (HR 1.46, $95 \%$ CI 1.01-2.12, $p=0.043$ ), while MMR deficiency was not (HR 1.04, $95 \%$ CI 0.48-2.22, $p=0.93$ ). No association with CSS was observed for both markers. High expression of MUC5AC showed a trend toward improved PFS (HR 0.44, 95 \% CI 0.18-1.07, $p=0.07)$, while no association of MMR deficiency with PFS was observed (HR 1.04, $95 \%$ CI 0.49-2.23, $p=0.92$ ). Again, no association with CSS was observed for both markers. Bivariate Cox regression analyses could not be performed for MUC6 due to low case numbers.
When only stage II and III patients were analyzed in bivariate Cox regression models, presence of MUC2 showed significant association with PFS (HR 2.05, $95 \%$ CI 1.29-2.35, $p=0.002$ ), while MMR deficiency did not (HR 1.12, $95 \% \mathrm{CI}$ $0.35-3.55, p=0.85)$. A trend toward shorter CSS was observed for presence of MUC2 $(p=0.07)$, but not for MMR deficiency $(p=0.95)$. High expression of MUC5AC was significantly associated with PFS (HR 0.13, $95 \%$ CI 0.02-0.91, $p=0.04)$, while no association of MMR deficiency with PFS was observed (HR 1.06, $95 \%$ CI 0.35-3.37, $p=0.92$ ). High MUC5AC expression showed a trend toward inferior CSS $(p=0.06)$, while no association of MMR deficiency $(p=0.89)$ with CSS was noted.

\section{Discussion}

Mucins have an important function as protective layer for epithelial tissues in the gut and elsewhere in the body. It is well known that during carcinogenesis mucins can be lost or aberrantly expressed in locations where they are not present constitutively. They might be involved in tumor progression and spread. However, the prognostic value of aberrant mucin expression in CRC is controversial $[5,6$, 9, 10].

In this study, we show that the loss of intestinal mucin MUC2 predicted adverse outcome, while the gain of gastric differentiation, as documented by aberrant MUC5AC and particularly MUC6 expression: was associated with favorable outcome. In addition, expression of MUC1 proved to be a marker of tumor progression and lymph node metastasis. A significant association between MUC1 expression and survival was, however, not detected in our cohort.

Previous publications have reported impact of MUC1 expression on tumor progression and also on survival [10, 20-24]. For instance, MUC1 expression has been related to higher TNM stage and reduced recurrence-free and overall survival in 206 patients with CRC [22]. Duncan et al. [23] found no association with $\mathrm{T}$ or $\mathrm{N}$ classification, but also reported a significant reduction of CSS for MUC1-positive tumors. Lugli et al. [10] analyzed a large number of tumors and observed, similar to our data, a positive association of MUC1 with T classification, but not with survival in MMR-proficient tumors. They also found no association with clinicopathological parameters in MLH1-negative cases. Hence, while MUC1 expression appears to be related to tumor stage, its impact on survival remains controversial.

MUC2 is a colonic mucin usually expressed by goblet cells. It is enriched in mucinous adenocarcinoma and can be lost during the carcinogenic process in conventional adenocarcinoma. Similar to MUC1, previous data about the prognostic impact of MUC2 are conflicting. In concordance with our results, Elzagheid et al. [25] found that the presence of 

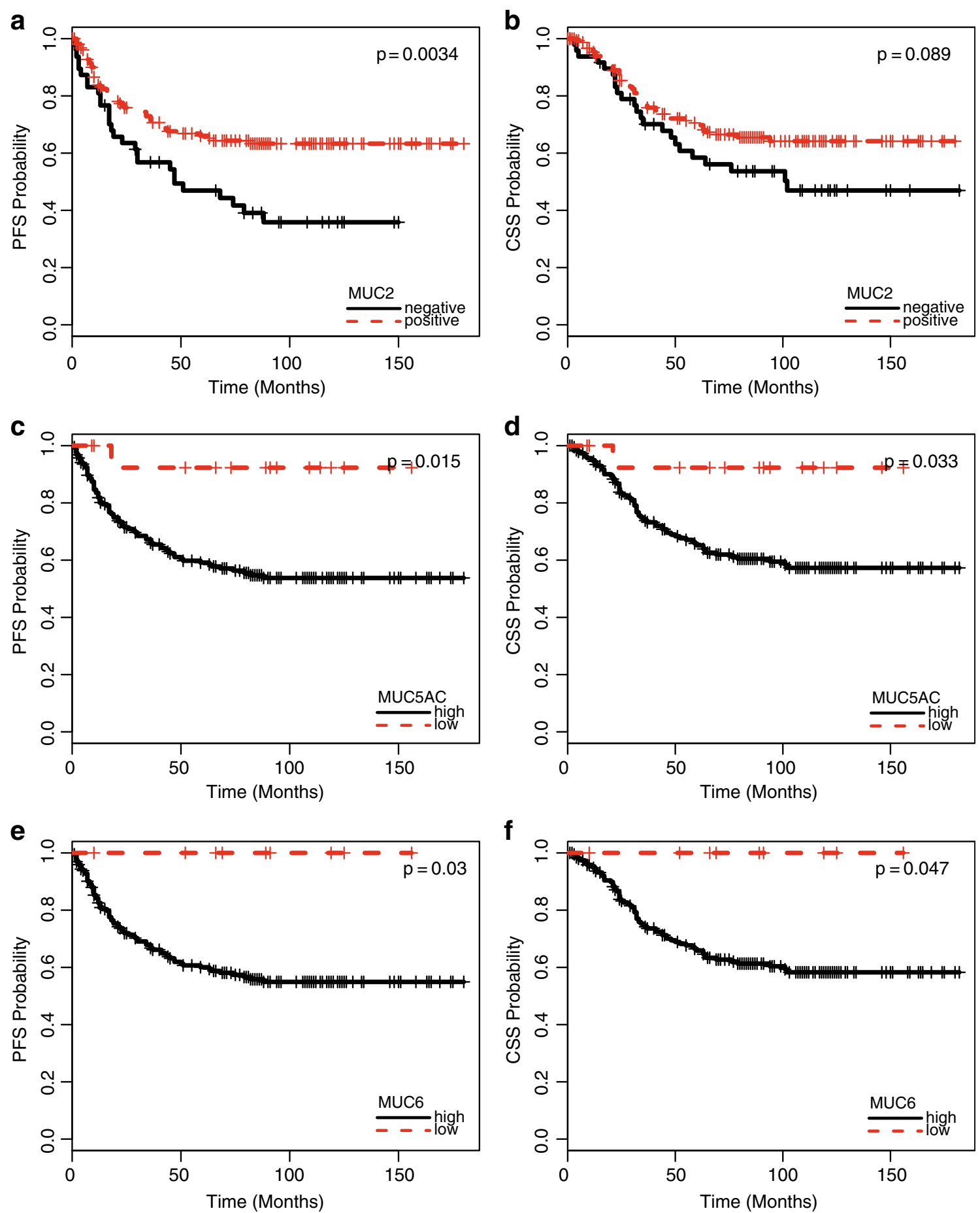

Fig. 4 Progression-free (a, $p=0.0034)$ and cancer-specific $(\mathbf{b}, p=0.089)$ survival of patients with stage II and III colorectal cancer related to the extent of MUC2 expression (present vs. absent). Progression-free (c, $p=0.015)$ and cancer-specific $(\mathbf{d}, p=0.033)$ survival of patients with

f

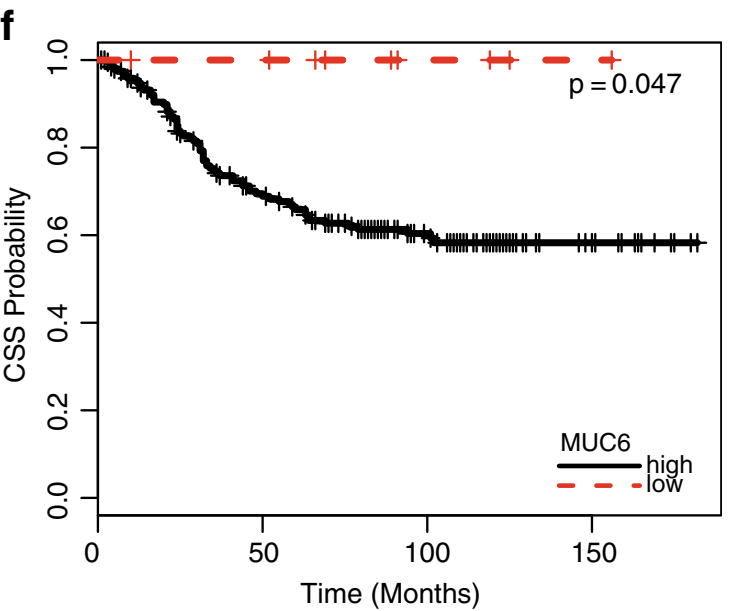

expression (high vs. low expression). Progression-free (e, $p=0.03$ ) and cancer-specific (f, $p=0.047$ ) survival of patients with stage II and III colorectal cancer related to the extent of MUC6 expression (high vs. low expression) stage II and III colorectal cancer related to the extent of MUC5AC

MUC2 significantly predicted longer disease-free survival and disease-specific survival in $141 \mathrm{CRC}$ patients across all stages. Accordingly, Lugli et al. [10] reported on a large set of 1420 cases that loss of MUC2 is associated with the presence of lymph node metastasis and with worse survival in both MMR- proficient and MMR-deficient tumors. In another study focusing on stages II and III CRC, Kang et al. [26] likewise noted significantly decreased overall survival when MUC2 expression was lost. However, other publications failed to identify a prognostic impact of MUC2 in CRC [20, 22, 24]. 
Literature data on the prognostic value of gastric type mucins MUC5AC and MUC6 in CRC are limited. Our study is the first to systematically analyze the prognostic impact of MUC6 expression in CRC. The expression of MUC6 is rare: We observed low expression of MUC6 in $27 \%$ and high expression of MUC6 in only $2 \%$ of patients. However, when MUC6 was present in the majority of cancer cells, affected patients had an excellent prognosis. Therefore, we propose that MUC6 (and MUC2) should be included in the panel of molecular markers applied in prospective studies aiming at risk stratification of CRC patients. Walsh et al. [27] identified MUC6 expression in $33 \%$ of CRCs. According to their and other literature data [28], MUC6 and MUC5AC expression is strongly associated with features of the serrated neoplasia pathway. The impact on outcome was not analyzed in that study. Interestingly, in vitro data generated in a study investigating pancreatic, colorectal, and breast cancer cell lines suggest that MUC6 may inhibit invasion of tumor cells through the basement membrane [29]. This may be a possible mechanism to explain why patients with tumors strongly expressing MUC6 have a favorable outcome.

The aberrant expression of MUC5AC in CRC was more frequent than that of MUC6 in our study. It predicted favorable outcome, and this effect was particularly strong in patients with stage II and III disease. In agreement with our data, a small study analyzing 41 cancer and 41 normal mucosa specimens as well as 21 metastatic lymph nodes also found that patients with tumors expressing MUC5AC had longer disease-free and of overall survival [30]. Another study reported a significant prognostic impact only in poorly differentiated adenocarcinoma, but not in well-differentiated or mucinoustype adenocarcinomas [31]. In a different study, MUC5AC was marginally associated with survival, but statistically significant only in stage IV disease. Hence, our data generally support the limited evidence from literature in that MUC5AC appears to be a significant, but moderately strong prognostic parameter in CRC.

MMR status was significantly associated with the expression of MUC2, MUC5AC, and MUC6, which is in concordance with literature data [27]. In MMR-proficient tumors, loss of MUC2 expression and high expressions of MUC5AC and MUC6 were associated with poor outcome, while no association with survival was detected in patients with MMR-deficient tumors. However, our cohort might not be suitable to prove the lack of impact of mucin expression in MMR-deficient tumors due to relatively small sample size in this subgroup of patients. In bivariate Cox proportional hazards regression models, we were able to confirm the prognostic impact of both MUC2 loss and MUC5AC overexpression in intermediate stages II and III, while MMR status had no impact on outcome. According to a previous study, lack of MUC2 was found to be an adverse prognostic factor in both MMR-proficient and MLH1negative CRC, but not in presumed HNPCC cases [10]. Data analyzing the prognostic impact of MUC5AC and MUC6, stratified by MMR status, have been lacking so far.

Divergent results in previous studies with respect to the prognostic impact of different mucins, especially MUC1 and MUC2, may be related to the methodology of assessment. We aimed to assess mucin expression in a systematic and easily reproducible fashion in a large number of CRC patients using a high number of tumor tissue cores. However, in other studies, different scoring systems have been used with different cutoffs $[10,20,22-24,26,30]$. Also, different antibodies are used with different staining protocols. Moreover, some authors have scored mucin expression specifically at the invasive margin $[21,32]$. This makes the comparison of studies difficult. Future (prospective) studies should aim to standardize the methodology of assessment, before testing whether MUC2, MUC5AC, and especially MUC6 expression can be used to stratify patients regarding therapy decisions in routine practice.

Our study has several other limitations. The analysis of microsatellite instability was performed only by immunohistochemical staining of MMR proteins, not by molecular analysis. Furthermore, there are general limitations of retrospective studies, which we tried to control by random selection of patients from a large institutional database and by adherence to strict exclusion criteria. Nonetheless, even considering these limitations, our data strongly suggest that loss of MUC2 as well as aberrant expression of MUC5AC and especially of MUC6 are relevant prognostic indicators in CRC.

In conclusion, we found loss of MUC2 expression to be a predictor of adverse outcome, while gain of aberrant MUC5AC and particularly MUC6 expression was associated with favorable outcome in CRC, especially in intermediate stages II and III. Further prospective studies evaluating adjuvant chemotherapy in stages II and III colon cancer should include MUC2, MUC5AC, and MUC6 expression analysis for patient stratification.

Acknowledgments Open access funding provided by Medical University of Graz.

Compliance with ethical standards Institutional Review Board approval was obtained from the Ethics Committee of the Medial University of Graz, Austria.

Conflict of interest The authors declare that they have no conflict of interest.

Open Access This article is distributed under the terms of the Creative Commons Attribution 4.0 International License (http:// creativecommons.org/licenses/by/4.0/), which permits unrestricted use, distribution, and reproduction in any medium, provided you give appropriate credit to the original author(s) and the source, provide a link to the Creative Commons license, and indicate if changes were made. 


\section{References}

1. Compton CC (2007) Optimal pathologic staging: defining stage II disease. Clin Cancer Res 13:6862s-6870s. doi:10.1158/1078-0432. CCR-07-1398

2. McLeod HL, Murray GI (1999) Tumour markers of prognosis in colorectal cancer. Br J Cancer 79:191-203. doi:10.1038/sj.bjc. 6690033

3. Lyall MS, Dundas SR, Curran S, Murray GI (2006) Profiling markers of prognosis in colorectal cancer. Clin Cancer Res 12: 1184-1191. doi:10.1158/1078-0432.CCR-05-1864

4. Corfield AP, Carroll D, Myerscough N, Probert CS (2001) Mucins in the gastrointestinal tract in health and disease. Front Biosci 6: D1321-D1357

5. Byrd JC, Bresalier RS (2004) Mucins and mucin binding proteins in colorectal cancer. Cancer Metastasis Rev 23:77-99

6. Bu X-D, Li N, Tian X-Q, et al. (2010) Altered expression of MUC2 and MUC5AC in progression of colorectal carcinoma. World $\mathrm{J}$ Gastroenterol 16:4089-4094

7. Jass JR, Walsh MD (2001) Altered mucin expression in the gastrointestinal tract: a review. J Cell Mol Med 5:327-351. doi:10.1111/j. 1582-4934.2001.tb00169.x

8. Lau SK, Weiss LM, Chu PG (2004) Differential expression of MUC1, MUC2, and MUC5AC in carcinomas of various sites: an immunohistochemical study. Am J Clin Pathol 122:61-69. doi:10. 1309/9R66-73QE-C06D-86Y4

9. Molaei M, Mansoori BK, Mashayekhi R, et al. (2010) Mucins in neoplastic spectrum of colorectal polyps: can they provide predictions? BMC Cancer 10:537. doi:10.1186/1471-2407-10-537

10. Lugli A, Zlobec I, Baker K, et al. (2007) Prognostic significance of mucins in colorectal cancer with different DNA mismatch-repair status. J Clin Pathol 60:534-539. doi:10.1136/jcp.2006.039552

11. Betge J, Pollheimer MJ, Lindtner RA, et al. (2012) Intramural and extramural vascular invasion in colorectal cancer: prognostic significance and quality of pathology reporting. Cancer 118:628-638. doi:10.1002/cncr.26310

12. Moertel CG, Fleming TR, Macdonald JS, et al. (1990) Levamisole and fluorouracil for adjuvant therapy of resected colon carcinoma. N Engl J Med 322:352-358. doi:10.1056/NEJM199002083220602

13. International Union Against Cancer U (2009) TNM classification of malignant Tumours, 7th edn. Wiley-Blackwell, West-Sussex

14. Bosman FT, Carneiro F, Hruban RH, Theise ND (2010) WHO classification of Tumours of the digestive system, 4th edn. IARC Press, Lyon

15. Jass JR, Love SB, Northover JM (1987) A new prognostic classification of rectal cancer. Lancet 1:1303-1306

16. Ueno H, Murphy J, Jass JR, et al. (2002) Tumour "budding" as an index to estimate the potential of aggressiveness in rectal cancer. Histopathol 40:127-132

17. Betge J, Kornprat P, Pollheimer MJ, et al. (2012) Tumor budding is an independent predictor of outcome in AJCC/UICC stage II colorectal cancer. Ann Surg Oncol 19:3706-3712. doi:10.1245/s10434012-2426-Z

18. Harbaum L, Pollheimer MJ, Kornprat P, et al. (2011) Keratin 7 expression in colorectal cancer-freak of nature or significant finding? Histopathol 59:225-234. doi:10.1111/j.1365-2559.2011. 03694.X

19. Lugli A, Tzankov A, Zlobec I, Terracciano LM (2008) Differential diagnostic and functional role of the multi-marker phenotype CDX2/CK20/CK 7 in colorectal cancer stratified by mismatch repair status. Mod Pathol 21:1403-1412

20. Baldus SE, Mönig SP, Hanisch FG, et al. (2002) Comparative evaluation of the prognostic value of MUC1, MUC2, sialyl-Lewis(a) and sialyl-Lewis(x) antigens in colorectal adenocarcinoma. Histopathol 40:440-449

21. Hiraga Y, Tanaka S, Haruma K, et al. (1998) Immunoreactive MUC1 expression at the deepest invasive portion correlates with prognosis of colorectal cancer. Oncology 55:307-319

22. Khanh DT, Mekata E, Mukaisho K-I, et al. (2013) Transmembrane mucin $\mathrm{MUC1}$ overexpression and its association with $\mathrm{CD} 10^{+}$myeloid cells, transforming growth factor- $\beta 1$ expression, and tumor budding grade in colorectal cancer. Cancer Sci 104:958-964. doi: $10.1111 /$ cas. 12170

23. Duncan TJ, Watson NF, Al-Attar AH, et al. (2007) The role of MUC1 and MUC3 in the biology and prognosis of colorectal cancer. World J Surg Onc 5:31. doi:10.1186/1477-7819-5-31

24. Manne U, Weiss HL, Grizzle WE (2000) Racial differences in the prognostic usefulness of MUC1 and MUC2 in colorectal adenocarcinomas. Clin Cancer Res 6:4017-4025

25. Elzagheid A, Emaetig F, Buhmeida A, et al. (2013) Loss of MUC2 expression predicts disease recurrence and poor outcome in colorectal carcinoma. Tumour Biol 34:621-628. doi:10.1007/s13277012-0588-8

26. Kang H, Min BS, Lee KY, et al. (2011) Loss of E-cadherin and MUC2 expressions correlated with poor survival in patients with stages II and III colorectal carcinoma. Ann Surg Oncol 18:711-719. doi:10.1245/s10434-010-1338-Z

27. Walsh MD, Clendenning M, Williamson E, et al. (2013) Expression of MUC2, MUC5AC, MUC5B, and MUC6 mucins in colorectal cancers and their association with the $\mathrm{CpG}$ island methylator phenotype. Mod Pathol 26:1642-1656. doi:10.1038/modpathol.2013. 101

28. Owens SR, Chiosea SI, Kuan S-F (2008) Selective expression of gastric mucin MUC6 in colonic sessile serrated adenoma but not in hyperplastic polyp aids in morphological diagnosis of serrated polyps. Mod Pathol 21:660-669. doi:10.1038/modpathol.2008.55

29. Leir S-H, Harris A (2011) MUC6 mucin expression inhibits tumor cell invasion. Exp Cell Res 317:2408-2419. doi:10.1016/j.yexcr. 2011.07.021

30. Kocer B, Soran A, Erdogan S, et al. (2002) Expression of MUC5AC in colorectal carcinoma and relationship with prognosis. Pathol Int 52:470-477. doi:10.1046/j.1320-5463.2002.01369.x

31. Imai Y, Yamagishi H, Fukuda K, et al. (2013) Differential mucin phenotypes and their significance in a variation of colorectal carcinoma. World J Gastroenterol 19:3957-3968. doi:10.3748/wjg.v19. i25.3957

32. Baldus SE, Mönig SP, Huxel S, et al. (2004) MUC1 and nuclear beta-catenin are coexpressed at the invasion front of colorectal carcinomas and are both correlated with tumor prognosis. Clin Cancer Res 10:2790-2796 